\title{
Unique Hemagglutination Activity of an Isolate of Newcastle Disease Virus
}

\author{
Kozo TAKASE ${ }^{1 *}$, Yasushi MURAKAWA ${ }^{1)}$, Sin-ichi ERIGUCHI ${ }^{1)}$ and Hideo FUJIKAWA ${ }^{1)}$ \\ ${ }^{1)}$ The Chemo-Sero-Therapeutic Research Institute, 1-6-1 Okubo, Kumamoto 860-8568, Japan
}

(Received 9 June 2000/Accepted 23 August 2000)

ABSTRACT. The MET95 strain of a lentogenic Newcastle disease virus (NDV) isolated from a broiler in Japan, showed unique hemagglutination (HA) activity. The MET95 strain failed to show HA when examined by rapid glass plate method although they showed HA titer of 1:1,024 by micro-plate method. This unique HA was also observed after the MET95 strain was passaged ten times in chickens. The failure of HA by rapid glass plate method was not shown in any other NDVs examined.

KEY WORDS: hemagglutination, Newcastle disease virus.

J. Vet. Med. Sci. 62(12): 1325-1326, 2000

Newcastle disease virus (NDV) of Paramyxoviridae has been well known to possess hemagglutination (HA) activity on the envelope [1]. This HA has been used widely so far for identification and titration of NDV [3]. In general, NDV grows well in embryonating chicken eggs with release of a high number of virus particles in allantoic fluids, even if a small number of NDV is inoculated. The virus-existence in allantoic fluids is easily confirmed by an HA test mixing the allantoic fluid with chicken erythrocytes. This HA test has been carried out by two methods: micro-plate or rapid glass plate method $[2,6]$.

In 1995, an NDV was isolated from the lung/trachea material of a 28-day-old broiler which had been reared in a flock with about 20,000 pen-mates which were not given any vaccines after hatching [4]. The isolate of NDV was cloned and named the MET95 strain. A characterization test revealed that the MET 95 strain has very low virulence to chickens; intracerebral pathogenicity for day-old chicks, intravenous pathogenicity of 6-week-old chickens, and mean death time of the minimum lethal dose of the MET95 strain were almost the same as those of the B1 strain [5].

However, we found that the HA of MET95 was different from B1 when examined by the rapid glass plate method. Namely, the MET95 strain failed to show HA when examined by rapid glass plate method but not by the micro-plate method. In this paper, this unique HA of MET95 is described compared with the other NDVs.

Eight strains of NDVs were used in the experiments. The MET95 strain was plaque-cloned three times in chicken kidney cell cultures and passaged four times in specific pathogen-free (SPF) embryonating chicken eggs after isolation. The MET95(p10) strain was obtained from the MET95 strain after 10 times of passage in SPF chickens. The B1 strain is a live vaccine strain which has been used worldwide. The Sato strain is a virulent strain which has been used as a challenge virus for vaccine efficacy tests of the national assay test in Japan. The Atami and Fuji strains are virulent NDVs derived

\footnotetext{
* Present address and correspondence to: TAKase, K. Department of Veterinary Medicine, Faculty of Agriculture, Kagoshima University, 1-21-24 Korimoto, Kagoshima 8900065, Japan.
}

from the Japanese Association of Veterinary Biologics (Tokyo). These NDVs were propagated in SPF chicken eggs and their allantoic fluids were used as virus material.

The VG/GA and La Sota clone 30 strains are vaccine viruses purchased from Merial Japan Ltd. (Tokyo) and Intervet K. K. (Tokyo), respectively. Each vaccine was rehydrated with the accompanying liquid diluent and used as virus material.

For red blood cell solution, chicken erythrocytes were obtained from 5-week-old SPF chickens, washed and re-suspended in phosphate buffered saline (PBS) to make a $5 \%$ or $0.5 \%$ suspension.

In the first experiment, virus materials of MET95, MET95(p10), B1 and Sato strains were diluted with PBS to make a serial two-fold dilution. Each diluted sample was examined for HA by the following two methods. HA titers were numerically recorded as the reciprocal of the highest dilution of samples of the HA expressed in each method.

Rapid glass plate method: One-tenth milliliter of each sample was placed on a glass plate. To that fluid was added $0.1 \mathrm{ml}$ of a $5 \%$ chicken erythrocyte solution. The two were immediately mixed with an applicator stick and HA was observed for one minute at room temperature $\left(20-25^{\circ} \mathrm{C}\right)$.

Micro-plate method: Fifty $\mu l$ of each sample was inserted into the wells of a 96-well micro-plate and a $0.5 \%$ chicken erythrocyte solution was added. The micro-plate was covered with a lid, gently swirled on plate-mixer, left at room temperature for $30 \mathrm{~min}$, and HA was observed.

In the second experiment, HA was applied to estimate fifty percent embryo-infective dose $\left(\mathrm{EID}_{50}\right)$. Each virus material was serially diluted 10 -fold in PBS and $0.1 \mathrm{~m} l$ of each dilution from $10^{-1}$ to $10^{-9}$ was injected into the allantoic cavity of 10-day-old SPF embryonating eggs. These eggs were incubated at $37^{\circ} \mathrm{C}$ for 3 days. Then, a small volume of allantoic fluid was removed and examined for HA by the two methods mentioned above (rapid glass plate method and micro-plate method). The HA-positive eggs were considered to be positive for virus infection and the $\mathrm{EID}_{50}$ was calculated.

Results are shown in Tables 1 (first experiment) and 2 (second experiment). Table 1 shows the HA titer contained in four virus materials when examined by the rapid glass 
Table 1. HA titer tested by rapid glass plate and micro-plate methods

\begin{tabular}{lcc}
\hline \multirow{2}{*}{ NDV strain } & \multicolumn{2}{c}{ HA titer } \\
\cline { 2 - 3 } & Rapid glass plate method & Micro-plate method \\
\hline MET95 & $<1: 1$ & $1: 1,024$ \\
MET95 (p10) & $<1: 1$ & $1: 1,024$ \\
B1 & $1: 16$ & $1: 1,024$ \\
Sato & $1: 16$ & $1: 1,024$ \\
\hline
\end{tabular}

plate or micro-plate methods. Every virus strain showed a high HA titer of 1:1,024 when observed by the micro-plate method. However, the MET95 and MET95 (p10) strains failed to show HA by the rapid glass plate method, while the B1 and Sato strains showed 1:16 of HA activity by this method. If the observation period of the rapid glass plate method was prolonged to $3 \mathrm{~min}$, the HA of the MET95 and MET95 (p10) strains was observed only slightly at a titer of $1: 1$.

Table 2 shows the $\mathrm{EID}_{50}$ titer of each virus material when the infection was determined by two HA testing methods. In the cases of the MET95 and MET95 (p10) strains tested by the rapid glass plate method, HA was not observed in any allantoic fluids of eggs even though the eggs were inoculated with low diluted virus materials. Their titers were expressed as less than $10^{2.5} \mathrm{EID}_{50} / \mathrm{ml}$. However, when tested by the micro-plate method, HA was positive in many eggs resulting in a high titer of $10^{7.9}$ and $10^{8.0} \mathrm{EID}_{50} / \mathrm{ml}$, respectively. On the contrary, the other strains showed no difference in HA results between the two methods, and each strain showed the same titer.

These results clearly showed the HA of the MET95 strain to be different from other NDVs. Although the mechanism is still not clear, the HA activity of the MET95 strain seems to be weak and the red blood cell agglutinates caused by the MET95 strain may be too small to see them by naked eyes.

In general, HA activity of viruses has been examined by the micro-plate method, and the rapid glass plate method can only be considered as giving an indication of the presence or absence of HA-positive agents. As shown in Table 2, the rapid glass plate method is reliable and useful when the presence of ordinary NDVs except for the MET95 and MET95 (p10) strains is tested. If we didn't do the rapid glass plate method and used the micro-plate method only in experiments, we couldn't find the difference of HA between the MET95 strain and other NDVs.

The weak HA of the MET95 strain was observed even after the virus was passaged ten times in vivo. In another experiment, we found that the pathogenicity of MET95 strain
Table 2. Egg-infective-titer determined by HA using rapid glass plate and micro-plate methods

\begin{tabular}{lcc}
\hline NDV strain & Rapid glass plate method & Micro-plate method \\
\hline MET95 & $\leq 2.5^{\text {a) }}$ & 7.9 \\
MET95 (p10) & $\leq 2.5$ & 8.0 \\
B1 & 8.9 & 8.9 \\
VG/GA & 6.5 & 6.5 \\
La Sota clone 30 & 7.0 & 7.0 \\
Sato & 8.1 & 8.1 \\
Atami & 7.5 & 7.5 \\
Fuji & 8.3 & 8.3 \\
\hline
\end{tabular}

a) $\log \mathrm{EID}_{50} / \mathrm{ml}$

to chickens was very low while the HI antibody titer of chickens infected with the MET95 strain was obviously higher than chickens infected with B1 strain of NDV [5]. Therefore, the MET95 strain may become a good candidate of vaccine strains for ND. In that case, vaccine virus of MET95 strain will be differentiated easily from other NDVs by HA using rapid glass plate method as shown in this study.

The MET95 strain was isolated from a broiler chicken of the field. This type of NDV might have been distributed already among chicken flocks. Attention has to be paid when HA tests are employed in characterization tests of NDV isolates.

The mechanism involved in weak HA activity of the MET95 strain remains to be determined and further studies are needed.

\section{REFERENCES}

1. Alexander, D.J. 1997. pp 541-569. In: Diseases of Poultry 10th ed. (Calnek, B.W. ed.), State University Press, Ames.

2. Beard, C.W. 1980. pp. 129-135. In: Isolation and Identification of Avian Pathogens 2nd ed. (Hitchner, S.B., Domermuth, C.H., Purchase, H.G. and Williams J.E. eds.), American Association of Avian Pathologists, Knett Square.

3. Hanson, R.P. 1980. pp. 63-65. In: Isolation and Identification of Avian Pathogens 2nd ed. (Hitchner, S.B., Domermuth, C.H., Purchase, H.G. and Williams J.E. eds.), American Association of Avian Pathologists, Knett Square.

4. Murakawa, Y., Takase, K., Murakami, T., Maeda, H. and Uchinuno, Y. 1998. J. Jpn. Soc. Poult. Dis. 34: 36-42.

5. Murakawa, Y., Takase, K., Sakamoto, K., Sueyoshi, M. and Nagatomo, H. 2000. Avian Dis. 44: 686-690.

6. Williams, J.E. 1980. pp. 136-140. In: Isolation and Identification of Avian Pathogens 2nd ed. (Hitchner, S.B., Domermuth, C.H., Purchase, H.G. and Williams J.E. eds.), American Association of Avian Pathologists, Knett Square. 